\title{
On Event Signal Reconstruction in Wireless Sensor Networks *
}

\author{
Barış Atakan and Özgür B. Akan \\ Next Generation Wireless Communications Laboratory \\ Department of Electrical and Electronics Engineering \\ Middle East Technical University, 06531, Ankara, Turkey \\ \{atakan, akan\}@eee.metu.edu.tr \\ http://www. eee.metu.edu.tr/ nwc/
}

\begin{abstract}
In Wireless Sensor Networks (WSN), the effective detection and reconstruction of the event signal is mainly based on the regulation of sampling and communication parameters used by the sensor nodes. The aim of this paper is to understand the effect of these parameters on the reconstruction performance of event signal in WSN. Theoretical analysis and results show that with proper selection of sampling and communication parameters, the event signal can be satisfactorily reconstructed at the sink. Furthermore, this study also reveals that the non-uniform and irregular sampling of the event signal outperform the uniform sampling in terms of the reconstruction performance while providing significant energy conservation. Moreover, it is also shown that node density is closely related with the quality.
\end{abstract}

Keywords: Wireless sensor networks, event signal reconstruction, non-uniform sampling, irregular sampling.

\section{Introduction}

In Wireless Sensor Networks (WSN), energy-efficient and reliable communication is mainly based on the regulation of sampling and communication parameters such as reporting frequency (or sampling frequency), number of source nodes, size of the source node selection area around the event. Therefore, to effectively reconstruct the observed physical phenomenon at the sink, it is imperative to understand the effect of these parameters over the reconstruction performance.

There has been some research efforts about the reconstruction of the observed phenomenon in WSN. In [2], the effect of the compression over the reconstruction of event signal is investigated. The upper bound for the compression rate which can achieve the given distortion bound is given. In [3], to effectively reconstruct

\footnotetext{
* This work was supported by the Turkish Scientific and Technical Research Council (TUBITAK) Career Award under grant KARIYER-104E043.

${ }^{1}$ The sampling frequency of a sensor node is the number of samples taken from the sensed event signal. However, since in WSNs each data packet includes a number of samples, reporting frequency which is defined as the number of transmitted data packet per unit time is directly proportional with sampling frequency. Therefore, throughout this paper, we mainly use the sampling frequency.
} 
the correlated observations of sensor nodes, both asymmetric and symmetric encoder settings are proposed. In [4, a source-channel matching approach is proposed to estimate the observed field, which can provide the analysis for the effect of the parameters such as number of nodes, power, field complexity over the estimation. In [5], to efficiently reconstruct the observed field, the tradeoff between coding rate and reliability characterized by the loss probability is investigated. In 6, the relation between the system throughput and reconstruction distortion is investigated for the case in which the reconstruction is performed by a large scale sensor network with mobile agents. However, none of these studies consider actual signal reconstruction. They use either estimated or compressed version of sensor samples to reach to an estimation of the event signal instead of an actual event signal reconstruction. Furthermore, they do not incorporate the sampling and communication parameters to investigate their effects on the actual reconstruction of the event signal.

In this paper, a theoretical analysis for the reconstruction of event signal at the sink is performed. The aim of this theoretical analysis is to understand the effect of the sampling and communication parameters over the performance of event signal reconstruction in WSN. The main contributions and results of this work can be outlined as follows:

1. Exact event signal reconstruction is performed using the sensor readings instead of an estimation of the event features. It is shown that with proper selection of sampling and communication parameters, an event signal can be satisfactorily reconstructed at the sink.

2. For a given application-specific reconstruction error constraint, the uniform sampling scheme, in which source nodes use the same sampling frequency, is inefficient for the reconstruction of the event signal with high frequency components. However, the non-uniform and irregular sampling schemes, in which source nodes use heterogeneous sampling frequencies, enable sensor network to effectively reconstruct the event signal with high frequency components as well as providing significant energy conservation.

3. The reconstruction performance can also be affected by the size of the source node selection area around the event. When this area is decreased, the lower reconstruction error can be obtained as well as significant energy conservation can be achieved with smaller number of sources. This highlights the node density problem coupled with the reconstruction process. That is, to achieve a certain level of reconstruction error, certain node density is imperative.

The remainder of this paper is organized as follows. In Section 2, we introduce the theoretical model for event signal reconstruction. In Section 3 , by using the theoretical model given in Section 2, we first give the results on the performance of event signal reconstruction with uniform, non-uniform and irregular sampling schemes, respectively. Then, we present the comparative results of these three sampling schemes. The concluding remarks are given in Section 4 . 


\section{Reconstruction of Observed Event Signal}

In WSN applications, the physical phenomena which are collectively observed by the sensor nodes can be modeled by a single point source or a field source. Here, we adopt the point source model [7] for event signal, and then we introduce the model for the event signal reconstruction. Note that, event signal reconstruction analysis introduced in this paper can be directly extended to the field source case.

\subsection{Modeling of Observed Event Signal}

In WSN applications, the event signal modeled by a point source is assumed to generate a continuous random process $f_{S}(t)$ having a variance $\sigma_{S}^{2}$ and a mean $\mu_{S}$. Assuming that the point source is at the center of the coordinate axis [7], the sensor node situated at location $(x, y)$ receives the signal given by

$$
f(x, y, t)=f_{S}\left(t-\frac{\sqrt{x^{2}+y^{2}}}{v}\right) e^{-\frac{\sqrt{x^{2}+y^{2}}}{\theta_{s}}}
$$

where $v$ denotes the diffusion velocity of $f_{S}(t)$ and $\theta_{s}$ denotes the attenuation constant of event signal $f_{S}(t)$. Since $f(x, y, t)$ is the delayed and attenuated version of $f_{S}(t)$, the variance and mean of $f(x, y, t)$ can be expressed by

$$
\begin{gathered}
\mu_{E}(x, y)=\mu_{s} e^{-\frac{\sqrt{x^{2}+y^{2}}}{\theta_{s}}} \\
\sigma_{E}^{2}(x, y)=\left(\sigma_{s} e^{-\frac{\sqrt{x^{2}+y^{2}}}{\theta_{s}}}\right)^{2}
\end{gathered}
$$

Using the observations which are attenuated and delayed version of the event signal, each sensor node generates its data packets. The $k^{\text {th }}$ data packet generated by sampling the received signal $f\left(x_{i}, y_{i}, t\right)$ by sensor node $n_{i}$ at location $\left(x_{i}, y_{i}\right)$, i.e., $S_{i, k}$, is defined as

$$
S_{i, k}=\left[f\left(x_{i}, y_{i}, t_{k p}\right) f\left(x_{i}, y_{i}, t_{k p+1}\right) \ldots f\left(x_{i}, y_{i}, t_{(k+1) p}\right)\right]
$$

where $S_{i, k}$ is $p \times 1$ vector, $p$ and $k$ denote the packet length and packet number, respectively, $f\left(x_{i}, y_{i}, t_{k p}\right)$ is a sample of event signal $f\left(x_{i}, y_{i}, t\right)$, taken at time $t_{k p}$. Next, we introduce the model for the reconstruction of event signal at the sink.

\subsection{Event Signal Reconstruction}

In WSNs, after the detection of an event, sensor nodes sample the event signal and send the generated data packets $\left(S_{i, k}\right)$ to the sink. During this process, sensor circuitries add noise to these packets $\left(S_{i, k}\right)$ as

$$
S_{i, k}^{\prime}=S_{i, k}+N_{i, k}
$$


where $S_{i, k}^{\prime}$ is the noisy version of the sensor packet $S_{i, k}$ and $N_{i, k}$ is the observation noise, where $N_{i, k} \sim N\left(0, \sigma_{N}^{2}\right)$. In addition to the additive noise, due to the constraint in cost, power, and communication, in WSNs, packet losses often arise during the communication between the sensor nodes and the sink. In this paper, to model the packet losses, we assume an overall packet loss probability $\lambda$ which includes loss due to channel errors, collision, and congestion. Hence, we define the lossy and noisy version of the sensor packet $\left(X_{i, k}\right)$ as

$$
X_{i, k}= \begin{cases}0 & \text { with probability } \lambda \\ S_{i, k}+N_{i, k} & \text { with probability }(1-\lambda)\end{cases}
$$

In WSNs, the packet losses heavily depend on the increasing contention in the forward path which is mainly based on the excessive communication load which results in collision, and congestion in the forward path. Therefore, the overall packet loss probability in a sensor network can be modeled by the sampling and communication parameters affecting the communication load over sensor network. Using ns-2 network simulator [11, we conduct comprehensive set of simulation experiments to model the overall packet loss probability $(\lambda)$ based on sampling and communication parameters used by sensor nodes. The simulation setting used in these experiments is given in Table 1, The experiment results given in Fig. 1 show that the overall packet loss probability $(\lambda)$ is mainly based on the number of source nodes $(M)$ and their sampling frequency $(f)$. Using OriginLab [10] data fitting toolbox, we analytically express the overall packet loss probability $\lambda$ as a function of number of source nodes and their sampling frequency as follows

$$
\lambda=1-e^{-0.01 f /\left(150 e^{-\frac{M}{15}}+5\right)}
$$

where $M$ is the number of source nodes, $f$ is the sampling frequency of source nodes (samples/sec.).

Since uncoded transmission considerably outperforms any approach based on the separation paradigm when the measured physical phenomenon is reconstructed within some prescribed distortion level [8], we assume that the sensors perform uncoded transmission. When the sensor nodes transmit the uncoded observations, minimum mean square error (MMSE) estimation is the optimal decoding technique. Therefore, the decoded version of the sensor packet $S_{i, k}$, i.e., $Z_{i, k}$, can be expressed as follows

$$
Z_{i, k}=\frac{\left(\sigma_{E}^{2}\left(x_{i}, y_{i}\right)+\mu_{E}^{2}\left(x_{i}, y_{i}\right)\right)\left(X_{i, k}\right)}{(1-\lambda)\left(\sigma_{E}^{2}\left(x_{i}, y_{i}\right)+\mu_{E}^{2}\left(x_{i}, y_{i}\right)+\sigma_{N}^{2}\right)}
$$

By using decoded sensor packets $\left(Z_{i, k} \forall i, k\right)$, to generate the samples used for reconstruction of the event signal $f_{S}(t)$, sink first averages the decoded sensor packets and then, arranges the successive averaged packets as follows

$$
A_{s}=\frac{1}{M}\left[\sum_{i=1}^{M} Z_{i, 1} \sum_{i=1}^{M} Z_{i, 2} \ldots \sum_{i=1}^{M} Z_{i,\left(\frac{\tau f}{p}\right)}\right]
$$




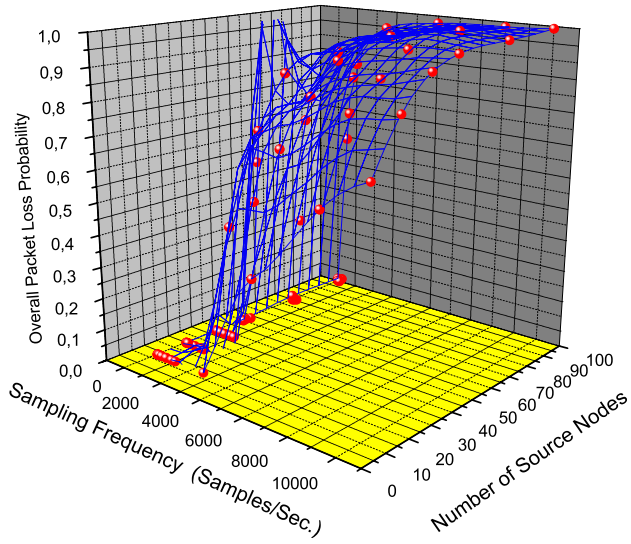

Fig. 1. The overall packet loss probability $(\lambda)$ for the varying $M$ and $f$

where $A_{s}(1 \times \tau f$ vector $)$ consists of the samples used for reconstruction of the event signal $f_{S}(t), \tau$ is a reconstruction interval (second). In order to obtain a reconstruction of the event signal $f_{S}(t)$, i.e., $\hat{S}(t), A_{s}$ is provided as the input to an ideal reconstruction (low-pass) filter 9] with frequency response $H_{r}(j \Omega)$ and impulse response $h_{r}(t)$. Then, the output of the filter is expressed as

$$
\hat{S}(t)=\sum_{n=1}^{\tau f} A_{s}[n] h_{r}\left(t-\frac{n}{f}\right)
$$

Here, assuming that the event signal $f_{S}(t)$ is a band-limited with the highest frequency component of $\Omega_{F}$, a cutoff frequency $\Omega_{c}$ of the filter must be $\Omega_{F}<$ $\Omega_{c}<\pi f-\Omega_{F}$ as long as $\pi f>2 \Omega_{F}$ is satisfied 2 Hence, for cutoff frequency $\pi f$, the corresponding impulse response $h_{r}(t)$ can be given by

$$
h_{r}(t)=\frac{\sin \pi f t}{\pi f t}
$$

and by substituting $h_{r}(t)$ into (9), we obtain $\hat{S}(t)$ as follows

$$
\hat{S}(t)=\sum_{n=1}^{\tau f} A_{s}[n] \frac{\sin [\pi(f t-n)]}{\pi(f t-n)}
$$

Using (7), (8), (9), (10), (11), the overall reconstruction operation can be formulated as in (12).

2 This is known as Nyquist Sampling Theorem and $\Omega_{F}$ and $2 \Omega_{F}$ are called as the Nyquist Frequency and Nyquist Rate, respectively. 


$$
\begin{aligned}
\hat{S}(t, \tau, f, M, p) & =\frac{1}{M} \sum_{k=1}^{\tau f / p} \sum_{j=1}^{p} \sum_{i=1}^{M}\left(\frac{\left(\sigma_{E}^{2}\left(x_{i}, y_{i}\right)+\mu_{E}^{2}\left(x_{i}, y_{i}\right)\right) X_{i, k}[j]}{\left(e^{-0.01 f /\left(150 e^{-\frac{M}{15}}+5\right)}\right)} \times\right. \\
& \left.\times \frac{\sin [\pi(f t-((k-1) p+j))]}{\left(\sigma_{E}^{2}\left(x_{i}, y_{i}\right)+\mu_{E}^{2}\left(x_{i}, y_{i}\right)+\sigma_{N}^{2}\right) \pi(f t-((k-1) p+j))}\right)
\end{aligned}
$$

Since the reconstructed event signal $\hat{S}(t, \tau, f, M, p)$ is a continuous time signal as the event signal $f_{S}(t)$, to measure the reconstruction efficiency, we resample $\hat{S}(t, \tau, f, M, p)$ and $f_{S}(t)$ with a sampling frequency which is higher than Nyquist rate. Based on the resampled version of $\hat{S}(t, \tau, f, M, p)$ and $f_{S}(t)$, we define the deterministic reconstruction error $E_{r}$ used for measuring the efficiency of the event signal reconstruction as

$$
E_{r}=\frac{1}{\tau f} \sum_{i=1}^{\tau f}\left(f_{S}[i]-\hat{S}[i, \tau, f, M, p]\right)^{2}
$$

where $f_{S}[i]$ and $\hat{S}[i, \tau, f, M, p]$ are $i^{t h}$ sample of $f_{S}(t)$ and $\hat{S}(t, \tau, f, M, p)$, respectively. Next, using the theoretical reconstruction model given in Section 2.2 we give the performance results of the event signal reconstruction with uniform, non-uniform and irregular sampling schemes.

\section{Reconstruction Performance Analysis and Results}

Here, we consider the scenario in which 300 sensor nodes are randomly deployed in a $100 \times 100 \mathrm{~m}$ field and a point source which characterizes the observed event signal is located at the center. We first perform the event signal reconstruction with three different sampling schemes:

- Uniform Sampling: In this scheme, source nodes sample the event signal with the same sampling frequency $(f)$.

- Non-uniform Sampling: In this scheme, source nodes select their sampling frequency according to their distance to the event location such that source nodes closer to event location use higher sampling frequency.

- Irregular Sampling: In this scheme, source nodes take samples from the event signal with a probability while sampling the event signal with a sampling frequency.

Then, we present the comparative results on the reconstruction performance of these sampling schemes. To make results more reliable, we take 100 Monte Carlo runs for every result in each scenario. For the event signal reconstruction process, we use $f_{S}(t)=\sin (2 \pi 50 t)+\sin (2 \pi 120 t)$ as an event signal which consists of $50 \mathrm{~Hz}$ and $120 \mathrm{~Hz}$ sinusoidal components as an event signal. According to the Nyquist Sampling Theory, since $f_{S}(t)$ includes $50 H z$ and $120 \mathrm{~Hz}$ sinusoidal components, $f_{S}(t)$ can be reconstructed with the sampling frequency larger than the Nyquist rate 240 Samples/sec. We set the simulation parameters in Table 1. 
Table 1. Simulation Parameters

\begin{tabular}{|c|c|}
\hline Network size & $(100 \times 100 \mathrm{~m})$ \\
\hline Attenuation constant $\left(\theta_{s}\right)$ & 50 \\
\hline Number of sensor nodes & 300 \\
\hline Number of source nodes & $16-100$ \\
\hline Reconstruction interval $(\tau)$ & $10 \mathrm{~s}$ \\
\hline Sampling frequency $(f)$ & $50-10000($ samples $/$ sec $)$ \\
\hline Packet length $(p)$ & $100($ samples $)$ \\
\hline Packet length $($ byte $)$ & 50 byte \\
\hline Sensor transmission range & $20 \mathrm{~m}$ \\
\hline Sensor channel capacity & $2 \mathrm{Mb} /$ sec \\
\hline Routing & DSR \\
\hline MAC & 802.11 \\
\hline
\end{tabular}

\subsection{Event Signal Reconstruction with Uniform Sampling}

In this section, we perform the reconstruction of event signal with the uniform sampling scheme. In Fig. 2, $E_{r}$ is given with varying $f$ for different values of $M$. As observed, for $f=50$ and $f=100, E_{r}$ takes its maximum value because the sampling frequencies 50 and 100 are less than the Nyquist rate (240 Samples / sec). However, although the sampling frequency 200 is less than the Nyquist rate, it can reduce $E_{r}$. This is mainly because that the sampling frequency 200 can be sufficient for the reconstruction of the $50 \mathrm{~Hz}$ sinusoidal component in $f_{S}(t)$, which decreases the overall reconstruction error. In Fig. 3 (a), the event signal $f_{S}(t)$ is shown. In Fig. 3 (b) the reconstructed event signal is shown by using the sampling frequency 500. Since the sampling frequency 500 is larger than the Nyquist rate, the event signal $f_{S}(t)$ can be satisfactorily reconstructed at the sink. The amplitude difference between $f_{S}(t)$ and the reconstructed signal results from the attenuation in the event signal from source nodes to sink.

The common technique for improving the reconstruction accuracy is to utilize oversampling. However, as observed in Fig. 2, it cannot be always possible to decrease $E_{r}$ by increasing $f$. While $f$ increases, as observed in Fig. 1, $\lambda$ increases. Therefore, at the higher $f$, the sufficient number of samples required for the reconstruction cannot be successfully delivered to sink and $E_{r}$ increases. This highlights the reconstruction problem for the event signal with high frequency components, which necessitates high sampling frequency according to the Nyquist Sampling Theory. Therefore, the uniform sampling does not stand as an effective sampling scheme to reconstruct the event signal with higher frequency components.

Reconstruction accuracy is also influenced by $M$ such that while $M$ increases, due to the increasing contention, $\lambda$ increases as shown in Fig. 1 Therefore, as $M$ increases, it is getting more difficult to deliver the sufficient number of samples to sink for the event signal reconstruction. Due to this result, as observed in Fig. 2, the smaller $E_{r}$ cannot be obtained at the higher $f$ by using higher $M$. However, as $M$ decreases, the smaller $E_{r}$ cannot be obtained at the lower $f$. 
Therefore, $M$ must be selected according to the event signal requirements such that if $f_{S}(t)$ has larger bandwidth, $M$ must be selected as smaller value and if $f_{S}(t)$ has smaller bandwidth, $M$ must be selected as higher value to obtain smaller $E_{r}$.

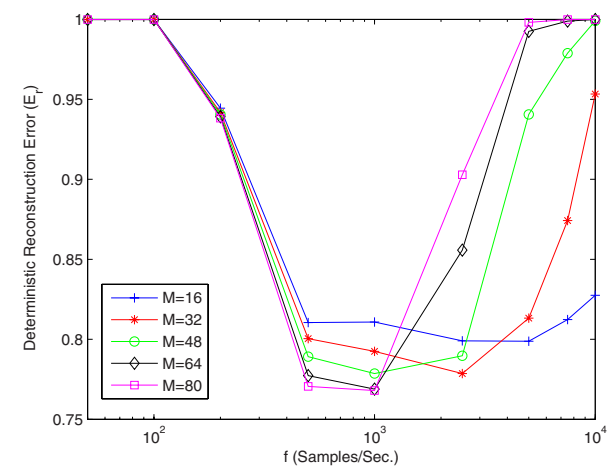

Fig. 2. $E_{r}$ with varying $f$ for different $M$ in uniform sampling

\subsection{Event Signal Reconstruction with Non-uniform Sampling}

Here, to realize non-uniform sampling, we divide $100 \times 100 \mathrm{~m}$ environment to 5 levels called $L_{1}, L_{2}, L_{3}, L_{4}$ and $L_{5}$ such that from $L_{1}$ to $L_{5}$, the size of the levels are $20 \times 20 \mathrm{~m}, 40 \times 40 \mathrm{~m}, 60 \times 60 \mathrm{~m}, 80 \times 80 \mathrm{~m}$ and $100 \times 100 \mathrm{~m}$, respectively. While the source nodes in $L_{1}$ use the sampling frequency $f$, the source nodes between $L_{1}$ and $L_{2}$ use $f / 2$ and the source nodes between $L_{2}$ and $L_{3}$ use $f / 3$ and so on. Consequently, there exist 5 different levels of sampling frequency from $f$ to $f / 5$.

In Fig. 4, $E_{r}$ is shown with varying $f$ for different $M$. For the illustration purposes, in $f$ axis of Fig. 4. we show only the largest sampling frequency in $L_{1}$. For example, $f=1000$ means that the source nodes in $L_{1}$ use $f=1000$ and the source nodes between $L_{1}$ and $L_{2}$ use $f=500$ and so on. Moreover, to compute $\lambda$, we use the average sampling frequency, $f_{a}$, which represents all sampling frequencies in 5 levels, i.e., $f_{a}=\left(f+\frac{f}{2}+\frac{f}{3}+\frac{f}{4}+\frac{f}{5}\right) / 5$.

As observed in Fig. 4, similar to uniform sampling, in non-uniform sampling, $E_{r}$ is not be reduced at the higher $f$. However, since $f_{a}$ is less than $f$ used in uniform sampling, as observed in Fig. 1, smaller $\lambda$ can be obtained at the higher sampling frequencies with respect to uniform sampling. This is mainly because decrease in $f$ results in less contention at the forward path. Therefore, at the higher $f$, non-uniform sampling enables WSN to deliver more information to sink and to obtain smaller $E_{r}$. This allows WSN to reconstruct the event signal with higher frequency components which necessitates the higher sampling frequency.

\subsection{Event Signal Reconstruction with Irregular Sampling}

In irregular sampling, source nodes take the samples from the event signal with the probability $\alpha$ while they sample the event signal with the sample frequency $f$. 


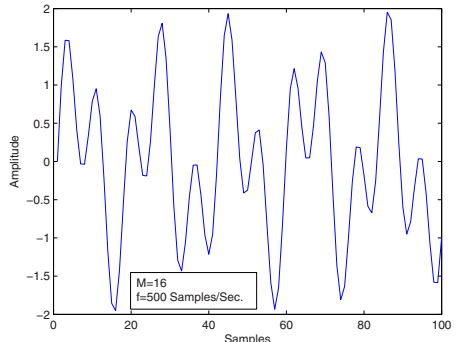

(a)

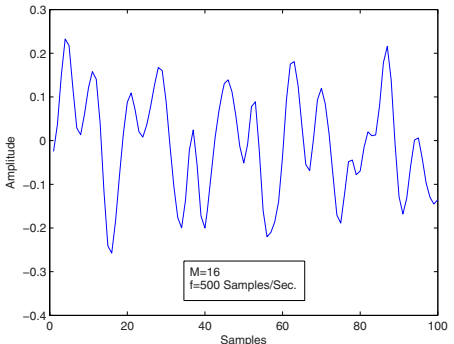

(b)

Fig. 3. (a) Event signal $f_{S}(t)$. (b) Reconstructed event signal.

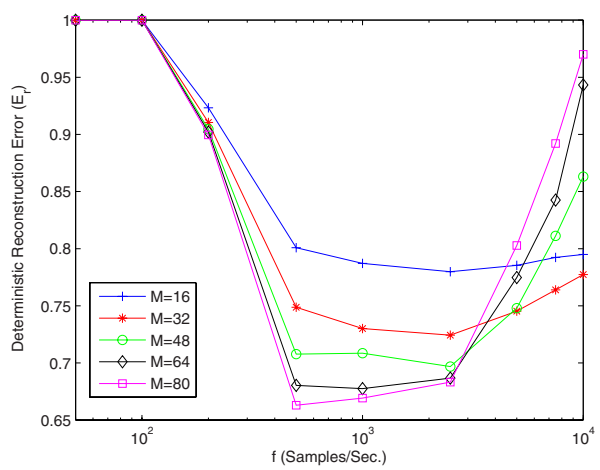

Fig. 4. $E_{r}$ with varying $f$ for different $M$ in non-uniform sampling

Therefore, in irregular sampling, to compute $\lambda$ we use $\alpha f$ as a normalized sampling frequency $f_{n}$.

To observe the effect of $\alpha$ on the event signal reconstruction, in Fig. 5, $E_{r}$ is shown with varying $f$ for different $\alpha$. It can be possible to choose $\alpha$ according to the reconstruction error requirements and the event signal bandwidth such that as $\alpha$ decreases, since $f_{n}$ decreases, less contention and overall packet losses in the forward path can be obtained. Therefore, it can be possible to deliver more information to sink at the higher $f$ and this enables the reconstruction of event signal with higher frequency components. However, for the reconstruction of event signal with low frequency components, as $\alpha$ decreases, since the number of delivered samples decreases, this results in increase in $E_{r}$. Therefore, to obtain lower $E_{r}$ at lower $f, \alpha$ must be increased.

\subsection{Comparative Results on Three Sampling Schemes}

To compare the uniform, non-uniform and irregular sampling schemes, in Fig. 6 $E_{r}$ is shown with varying $f$ for these three sampling schemes. In terms of $E_{r}$, the non-uniform sampling scheme outperforms the uniform and irregular sampling 


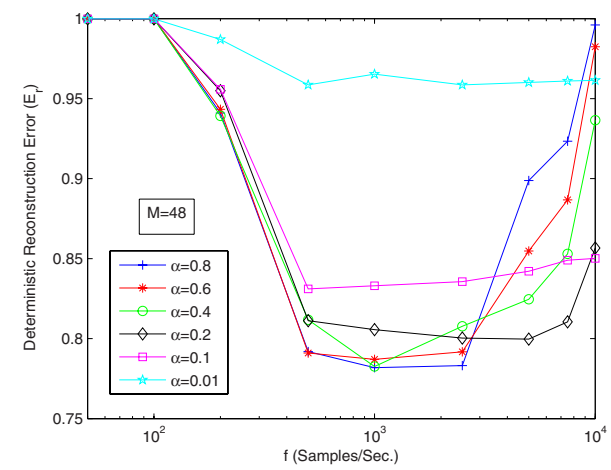

Fig. 5. $E_{r}$ with varying f for different $\alpha$

scheme. For an application-specific $E_{r}$ constraint $\left(E_{r}=0.85\right)$ shown in Fig. 6] non-uniform sampling scheme enables the sensor network to reconstruct the event signal having higher frequency components as well as providing smaller $E_{r}$ with respect to non-uniform and irregular sampling. For smaller $f$, the uniform and irregular sampling have almost the same $E_{r}$ performance.

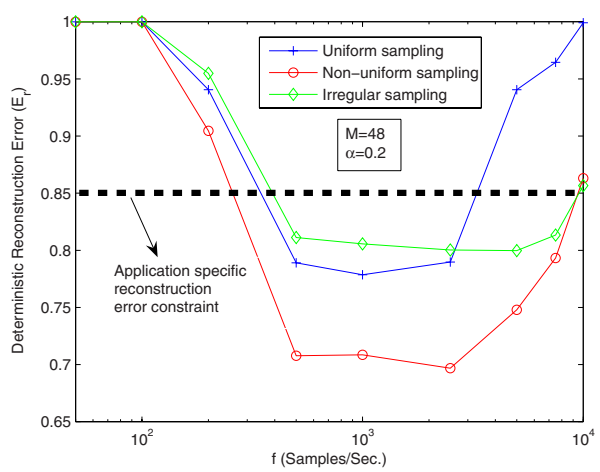

Fig. 6. $E_{r}$ with varying $f$ for three reconstruction schemes

However, for higher $f$, irregular sampling provides smaller $E_{r}$ performance with respect to uniform sampling scheme. Therefore, with a given applicationspecific $E_{r}$ constraint, it can be possible to reconstruct the event signal having higher frequency components using irregular sampling scheme.

To show the energy efficiency of three sampling schemes, we evaluate the average energy consumption for the three reconstruction schemes. We assume that each source node consumes one unit of average energy denoted by $E_{a v}$ to transmit a sample of the event signal to sink. Using the same simulation parameters $(M=48, \alpha=0.2, \tau=10 \mathrm{~s})$ in Fig. 6. we can compute the average energy consumption as $\tau f M E_{a v}$. For all sampling schemes, $\tau, M$ and $E_{a v}$ are 


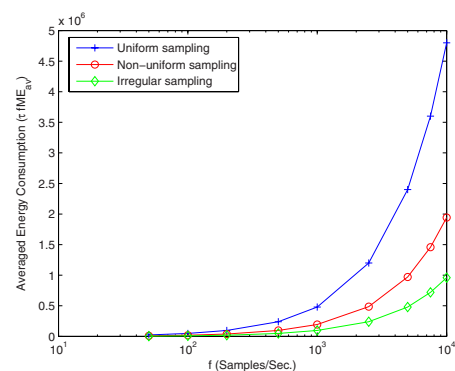

(a)

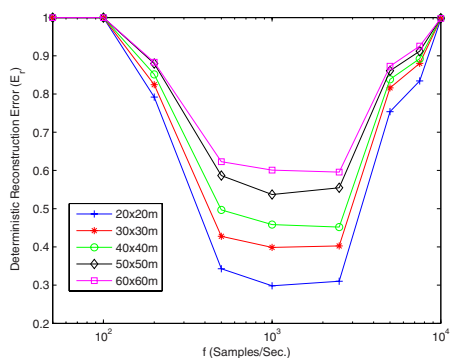

(b)

Fig. 7. (a) Average energy consumption for three sampling schemes. (b) $E_{r}$ with varying $f$ for different size of $A$.

the same. However, $f$ changes according to the sampling schemes such that while in the uniform sampling each source node transmits $f$ samples per second, in irregular sampling, each source node transmit $f_{n}=\alpha f$ samples per second and in the non-uniform sampling, on average, $f_{a}$ samples per second. According to this energy model, in Fig. 7 (a), for three sampling schemes, the average energy consumption is shown with varying sampling frequency. The irregular and nonuniform sampling significantly outperforms the uniform sampling in terms of energy consumption, because the irregular sampling and the non-uniform sampling transmit the less number of samples per unit time $\left(f_{n}\right.$ and $f_{a}$, respectively $)$ to reconstruct the event signal with respect to the uniform sampling.

The reconstruction error can also be affected by the attenuation in the received event signal. The attenuation in the received signal increases with the distance between the point source and the source nodes. In Fig. 7 (b), for the different size of source node selection area $(A)$ around the event and for $M=48$, the reconstruction error is shown with varying sampling frequency by using the uniform sampling scheme. The reconstruction error $\left(E_{r}\right)$ decreases while $A$ decreases because the attenuation in the received signal decreases while $A$ decreases. Furthermore, it is seen in Fig. 7 (b) that the minimum $E_{r}$ is achieved for $A=20 x 20 m$ and $M=48$. Therefore, to achieve this minimum reconstruction error, in each $10 x 10 \mathrm{~m}$ area, there should exist at least 12 sensor nodes. This highlights the node density problem in the reconstruction process such that for a certain level of reconstruction error, a certain node density is imperative.

\section{Conclusion}

In this paper, theoretical analysis and results show that with proper selection of sampling and communication parameters, the observed physical phenomenon can be satisfactorily reconstructed at the sink node. It is also shown that the uniform sampling is inefficient for the reconstruction of the event signal with high frequency components and non-uniform sampling and irregular sampling significantly outperform the uniform sampling for the reconstruction of event sig- 
nal with high frequency components and energy conservation. Therefore, unlike the existing congestion control mechanisms, non-uniform and irregular sampling based rate control stands as a promising approach. Furthermore, as a result of the attenuation in the sensed event signal, it is also shown that to achieve a certain level of reconstruction error, certain node density is imperative.

\section{References}

1. I. F. Akyildiz, W. Su, Y. Sankarasubramaniam, E. Cayirci: Wireless Sensor Networks: A Survey. Computer Networks Journal (Elsevier) vol. 38, Issue 4, (2002) 393-422

2. A. Kashyap, L. Alfonso, L. Monta, C. Xia, Z. Lui: Distributed Source Coding in Dense Sensor Networks In Proc. IEEE DCC (2005)

3. S. S. Pradhan, K. Ramchandran: Distributed Source Coding: Symmetric Rates and Applications to Sensor Networks. In Proc. IEEE DCC (2000) 363-372

4. W. Bajwa, A. Sayeed, R. Nowak: Matched Source-Channel Communication for Field Estimation in Wireless Sensor Networks. In Proc. IPSN'05 (2005) 332-339

5. D. Marco, D. L. Neuhoff: Reliability vs. Efficiency in Distributed Source Coding, In Proc. IPSN'04, (2004)

6. M. Dong, L. Tong, B. M. Sadler: Source reconstruction via mobile agents in sensor networks: throughput-distortion characteristics In Proc. IEEE MILCOM 2003, Boston, USA, (2003)

7. M. C. Vuran, O. B. Akan: Spatio-temporal Characteristics of Point and Field Sources in Wireless Sensor Networks In Proc. IEEE ICC 2006, stanbul, June (2006)

8. M. Gastpar, M. Vetterli, Source-Channel Communication in Sensor Networks: In Proc. IPSN'03, Palo Alto, USA, (2003)

9. A. V. Oppenheim, R. W. Schafer, J. R. Buck: Discrete-Time Signal Processing Prentice Hall, 1999.

10. http://www.originlab.com/

11. The Network Simulator, ns-2, http://www.isi.edu/nsnam/ns/ 